\title{
Diseases of the Musculoskeletal System in the Elderly
}

\author{
Iliadis Christos ${ }^{1}$, Monios Alexandros ${ }^{2}$, Frantzana Aikaterini ${ }^{1}$, Taxtsoglou Kiriaki ${ }^{3}$ and Kourkouta Lambrini ${ }^{1}$ \\ 1. Department of Nursing, Alexander Technological Educational Institute of Thessaloniki, Thessaloniki 57400, Greece \\ 2. 7th Gymnasium of Athens, Athens 57400, Greece \\ 3. University Orthopaedic Clinic General Hospital "C. Genimatas" of Thessaloniki, Thessaloniki 54635, Greece
}

\begin{abstract}
Old age is a part of life; it is of major importance due to the fact that it affects all human beings. It is necessary that substantial knowledge regarding diseases with increasing frequency such as diseases of the musculoskeletal system in the elderly to be acquired by doctors and nurses as well, in order these disorders to be prevented properly. Diseases of the musculoskeletal system cause noticeable damage to old people. As a result, these disorders can cause reduced molity and even death.
\end{abstract}

Key words: Diseases, elderly, muscoloskeletal system.

\section{Introduction}

Normal aging is considered to be the harmonious geratic development and decline in the body's tissues and organs. On the other hand, premature senescence is characterized as the asynchronous, disharmonious and premature senile decline in the body's tissues and organs. This decline happens because of specific causes of nosology, they appear before the age of sixty. The decline in cognitive function seems to be due to cognition rather than aging. Verbal skills are increasing over time and the activity of obtaining knowledge is continuous through life as we notice lots of senior people to attend universities and their performance is excellent. Ancient societies might interpret old age in a very simple way. It was due to the attitude related to the gradual exhaustion of the individual's natural strength. A human body is gradually depleted like the depletion of the oil in an oil lamp, which is constantly burning and lighting. Some societies that were more sophisticated than other ones paralleled the age with seasons of the year. The sense of aging was always accompanied by depressed mood since it contained the irrevocable stage of the process of life that leads to

Corresponding author: Kourkouta Lambrini, Ph.D., professor, research field: history of nursing, ethics \& deontology. E-mail: laku1964@yahoo.gr. death. Human beings are complicated organic complexes who deteriorate and grow old over the passage of time. The way an individual ages depens on lots of variables such as heredity, living conditions, nutrition, health status and others. [1] Nutrition plays an important role in maintaining good health and it is also packed with social, cultural and symbolic meanings. [2] Food and water are considered as human care and are essential to life [3].

The cultural, financial, social and environmental changes have contributed to the increased percentage of the elderly population, mainly in developed countries worldwide. According to the UN (United Nations), there is no definite age limit; it is agreed that people over sixty are integrated into the category of the third age. Simultaneously, it is observed that as time goes by and across countries, this limit has upward trends. The designation of the limits of the old age is difficult to be specified as life expectancy is different amongst countries. Specifically, the limit of the old age in Africa is lower than the one in Western countries, for the healthcare system and the standard of living regarding the elderly is worse. The key feature of old age related to the musculoskeletal system is the progressive decrease in muscle mass. The mobilization, which is restricted, many times, in the old age is a factor that helps the development of muscular atrophy. 
[4] Bones are very brittle and a slight knee flexion and kyphosis, wrist and hip flexion are noticeable. Furthermore, it seems there are smaller vertebrae, thinner intervertebral discs. It is also observed decline in bone mass, and finally, decrease of bone minerals. [5]

In this paper, the study material was based on recent articles, at least, the latest five-year related articles that were extracted mainly from the international database Medline and Hellenic Academic Libraries (HEAL-Link). The keywords that were used were: musculoskeletal system, old age. An exclusion criterion of articles was the language, except for Greek and English.

The diseases described in this study are the most frequent. They have a serious impact on the quality of older patients' lives.

The purpose of this retrospective study is to highlight that the study of the diseases of the musculoskeletal system in the elderly is of major importance. Thus, health professionals are able to recognize, prevent and treat older people's symptoms. In fact, nurses can undoubtedly offer generously their nursing training in order this vulnerable age to be generally supported.

\section{Osteoperpsis}

Osteoporosis is a disease in which bones become fragile due to the loss of bone mass. Osteoporosis can manifest itself at any age. It is estimated that $80 \%$ of patients suffering from osteoporosis are women. There are lots of factors that increase the risk for bone loss and osteoporosis. Some of these factors are preventable and thereby, the risk of the disease can be removed. However, this is impossible for other factors. Some factors that are not able to be prevented are: gender (women are affected by osteoporosis more often than men), age (the older the person, the higher the risk of osteoporosis), stature (short and slim women run a higher risk than others), family history (family predisposition), origin (white women and those of
Asian origin run a greater risk of being affected by this disease). [6]

There are other factors that increase the risk for osteopenia and osteoporosis. These factors are: hormones (low blood estrogen levels because of abnormalities of the menstrual cycle or menopause are causes that lead to osteoporosis in women. On the other hand, men run a higher risk of being affected by osteoporosis when blood testosterone is low), anorexia nervosa (an eating disorder), inadequate dietary intake of calcium and vitamin $\mathrm{D}$, medicines that increase the risk of osteoporosis such as corticosteroids, decreased physical activity, sedentary lifestyle and prolong bed rest. Not only does smoking harm the heart and lungs, but also the human bones. Also, excessive alcohol consumption leads to weakened bones, and as a result, to bone loss. [7]

Regarding the prevention and treatment of osteoporosis, a diet rich in calcium and vitamin D is essential. Furthermore, regular physical activity and a healthy lifestyle are of major importance. [8]

The prevention of osteoporosis includes:

- A diet rich in calcium: low-fat dairy products (yoghurt, milk cheese), foodstuffs enriched with calcium (orange juice, cereal, bread) are some of proper dietary sources of calcium.

- Vitamin D: sun exposure is necessary in order the human body to synthesize vitamin D. Vitamin D helps the intestine absorb calcium and it strengthens human bones. Foods rich in vitamin D include fish, liver, egg yolk and dairy products.

- Regular physical activity such as walking, cycling, jogging, going up and down stairs, weightlifting, dancing and other sports.

A healthy lifestyle. Smoking and excessive alcohol intake should be avoided. [9]

\section{Rheumathoid Arthritis}

Rheumatoid arthritis (RA) is a chronic systemic autoimmune disease whose main characteristic is persistent peripheral joint inflammation. It usually 
affects symmetrically the joints of the hands and feet.

In most cases, rheumatoid arthritis (RA) gradually starts to influence different joints. These joints are affected in a symmetrical way. If a joint is affected on one side of the body, the corresponding joint on the other side of the body will be affected, too. Rheumatoid arthritis (RA) initially affects the small joints in people's hands, feet, fingers, toes, wrists elbows and ankles. The affected joints cause pain and stiffness. They are swollen and deformed; there is also joint anchylosis. Wrists may be swollen. Should knees be affected, cysts may be present. If these cysts are ruptured, they cause pain and swollen legs. [10]

The purpose of treatment is to combat inflammation and relieve patient's pain, maintain mobility and functionality of joints and avoid their deformation. First and foremost, the joints must be relaxed. Splints may be used to immobilize a joint to relax. However, there seems to be a need of joint mobilization; a physiotherapist can help the patient perform mild exercises in order the latter to avoid muscle stiffness and maintain mobility and muscle strength. Swimming and hot water baths might be very helpful. The patient's can do exercises underwater to improve their condition. Patients ought to eat high fat foods, for they can reduce the inflammation to a small extent. Nevertheless, patients should avoid a high-red meat diet. It is essential the patient and his/her family be informed and educated by a group of professionals who are responsible for taking care of the patient. [11]

\section{Sarcopenia}

Using the term sarcopenia, we refer to the progressive and generalized loss of skeletal muscle mass and strength. This loss is increasing gradually as the person grows older. Sarcopenia is associated with the limitation of body's function and the increase of old age people's disability. [6]

The onset of sarcopenia may be of unknown etiology. However, its gradual progress is related to physical disability, poor quality diet, restriction of physical activity, changes regarding protein metabolism and reduced anabolic ability of the human organism to digest dietary proteins and synthesize muscle proteins (anabolic resistance). This decrease in muscle mass in the elderly is associated with serious diseases. Thus, it is a modern scourge and people must start prevent themselves from sarcopenia even before they reach the age of forty. [12]

As regards the handling and treatment of sarcopenia, the dietary management is based mainly on adequate protein intake. Amino acid supplementation per os has the potential to reduce the loss of muscle mass during patient's immobility, prevent sarcopenia and increase the ability and speed of patients' gait. Regarding nutrition, it is vital an old age patient have meat and animal proteins, owing to the fact that they increase the synthesis of anabolic substances. The supplemental administration of other nutrients such as creatine and vitamin D has a positive impact on people already diagnosed with sarcopenia. The supplemental intake of creatine increases muscle strength especially when it is combined with proper exercise, while it does not increase muscle mass. Specifically, administration of calcium and vitamin D is highly recommended to women after menopause. Exercise, mainly in the form of aerobic exercise 2-3 times per week or even simple routine agility exercises, a balance and strength training workout when possible is an important therapeutic agent for people suffer from sarcopenia. [13]

\section{Fractures}

Fractures are broken bones which are broken completely or partially in each body area and in any way. The older the person is, the more possibilities of a fractured bone he has. Frequently, fractures have been causes of death of an old individual. Sometimes these fatal fractures are not considered as important. Therefore, the patient and his family surroundings do not pay special attention to it. [14]

The main symptoms are evident in the area around 
the fracture. There is a lot of pain and the area is swollen. A bone can be deformed or even the fractured bone may puncture the skin. As a result, the area is painful while there is redness and mild swelling. [15]

The treatment of fractures includes the use of a cast or removable brace, plates, internal splints, screws, wires or other devices in order the fractured bone to be kept in the right position during its healing (porosis) of the fractured bone.

In fact, there are two types of treatment:

- External fixation methods. They include plaster bandages or fracture bracing, and other devices

- Internal stabilization methods. They include metal plates with intramedullary nails with distal screws and other devices. They hold the broken parts of the fractured bone in the appropriate position till the bone is healed. [16]

\section{Falls}

As people grow older, they experience changes regarding their vision and hearing. Their postural reflexes are disturbed and there is reduced ability for them to stand after sitting or lying down. The reasons above make the elderly be prone to falls. The older the individual, the more frequent the falls are. These falls may be causes of death. [17]

The main risk factors for falls amongst the elderly are divided into two categories: endogenous and exogenous. The endogenous risk factors are the following: age (people over 65 are most susceptible to falls), reduced mobility (unsteady walking), person's health condition, sensory changes (deterioration of hearing or vision), the reduced mental health (dementia, problems with orientation). The exogenous factors are the following: medicine (polypharmacy, drug toxicity and psychotropic drugs), environmental risks and recent hospitalization. [18]

Regarding prevention, the regular physical activity improves strength and muscle tone, as it maintains the flexibility of joints, tendons and ligaments. All the rooms in the house must be kept clean without many small objects on the floor. So, it is provided better mobility and security. A lot of lighting in living spaces, a permanently turned-on lamp during the night, "handles" in the bathrooms and stairs to support the elderly are recommended. [19]

\section{Conclusion}

As people grow older, they experience changes. The human organism deteriorates not only physically but also mentally. Muscles tend to lose their strength as muscle atrophy appears. Joints are observed to lose their stability and agility. The whole area of the joint and range of motion is reduced. Particularly, the joints of the hip and knee are mostly affected. As regards bones, osteoporosis is observed. Reduction of the bone mass characterizes osteoporosis. As a result, the strength of the bone, as well as its content in minerals is reduced and, thus, the risk of a fracture is on the increase. [20, 21]

Support and improvement of the needs amongst the elderly is one of the key priorities of the society. This can be achieved with the aid of organizations and private, public or voluntary clubs in collaboration with municipalities and other local authorities. All these organizations contribute to promoting health and well being, since they provide various projects providing exercise, recreation, creation, participation in public affairs and continuous vigour according to people’s age. [22]

The role of both the nurse and physician is decisive for older people suffering from a musculoskeletal problem. In other words, these professionals have the proper knowledge to support and encourage the elderly for their speed recovery in order to improve their lives.

\section{References}

[1] Kastenbaum, R., 1982. "Love and Intimacy in the Third Age.” In The Third Age. Athens: Psychogios, 81-9.

[2] Tsaloglidou, A., Rammos, K., Kiriklidis, K., Zourlandani, A., and Matziari, C., 2007. "Nurses' Ethical Decision-Making Role in Artificial Nutritional Support." BJN 16 (16): 996-1001. 
[3] Tsaloglidou, A., Rammos, K., Kyparos, A., Dimitriadou, A., and Matziari, C., "Ethical Issues in Withholding or Withdrawal of Artificial Nutrition and Hydration.” IJCS 1 (2): 66-73.

[4] Koukourikos, K., Tsaloglidou, A., and Kourkouta, L. "Muscle Atrophy in Intensive Care Unit Patients.” Acta. Inform. Med. 22 (6): 406-10.

[5] Christodoulou, C., and Kontaxakis, B. 2000. The third age. Athens: Greek.

[6] Athanatou, E., 2007. Medical and Surgical Clinic Nursing, 7th ed. Athens: The Tavitha.

[7] Karponis, A.

2004. “Osteoporosis-Epidemiology-osteoporotic Fracture." Bone 15 (1): 25-6.

[8] Mccormick, D. B. 1989. "Two Interconnectes B Vitamins: Riboflavin and Pyridoxine.” Physiological Reviews 69 (4): 1170-98.

[9] Kotzampasakis, S., and Christopoulou, I., 2005. "Education for the Prevention and Treatment of Osteoporosis: The Nursing Role.” NURSING 44 (1): 57-62.

[10] Archimandritis, I., 2010. Internal Pathology. Athens, Greek.

[11] Mpoki, K.A., 2001. "Biological Therapies in Rheumatoid Arthritis.” MEDICINE 80 (4): 328-33.

[12] Evans, W. J., 1985. "What is Sarcopenia?” J. Gerontol A Biol. Sci. Med. Sci. 50: 5-8.
[13] Baumgartner, R. N., Koehler, K. M., Gallagher, D., Romero, L., Heymsfield, S. B., Ross, R. R., Garry P. J., and Lindeman, R. D., 1998. "Epidemiology of Sarcopenia among the Elderly in New Mexico.” Am. J. Epidemiol. 147: 755-63.

[14] Perry, C., Elstom, J., and Pankovich, A., 1999. The Manual of fractures. Athens: Publications Parisianou.

[15] Grosomanidou, B., Zouka, M., and Vassilakos, D., 2002. “Orthopaedic Injuries.” Topics of Anaesthesiology and Intensive Medicine 12: 168-72.

[16] Bekridelis, A., 1999. "Multiple Trauma." Topics of Anaesthesiology and Intensive Medicine 9 (18): 122-31

[17] Keramiotou, K., 2010. "Falls and Natural Environment in the Elderly-Risk Factors and Occupational Therapy." Occupational Therapy (41): 19-25.

[18] Morakis, A. 2015. "Risk Factors for Falls in the Elderly.” http://www.andreasmorakis.gr. Posted on 21.02.2015.

[19] Patistea, E., and Androutsopoulou-Litra, K., 2007. "The Prevention of Falls as an Important Aspect of a Healthy and Successful Very Old Age” Nursing 46 (4): 468-83.

[20] Chatzimpougias, I. 2002. Anatomic Human Terms. Athens: Publications GM.

[21] Tsaousoglou, A., and Koukourikos, K., 2007. "Quality and Health Services.” Stigma 15 (1): 18-24.

[22] Needham, J. 2004. Gerontological Nursing Care. Translated and edited by Karachalios, C. Athens: Elli. 